\title{
On the Application of Some Interpolating Functions in Physics*
}

\author{
A. J. Jerri**
}

(April 29, 1969)

J. M. Whittaker considered interpolating equidistant samples by using the cardinal series. In this paper we consider the extension provided by the generalized sampling theorem of Kramer for nonequidistant samples. We calculate some sampling functions for cases of interest in mathematical physics. In particular, this includes the $S$-matrix contribution due to Regge poles, especially when a series expansion other than that of Fourier-Legendre type is needed.

Key words: Cardinal series; interpolation; Regge poles; special functions.

\section{Introduction}

J. M. Whittaker $[1]^{1}$ considered the interpolation of functions sampled at equidistant points. This resulted in his cardinal series and its integral representation for the functions as finite Fourier transforms. The result was introduced to Communications Theory by Shannon [2] in the form: if

$$
f(t)=\int_{-a}^{a} e^{i x t} g(x) d x
$$

where

$$
\begin{gathered}
g(x) \epsilon L^{2}(-a, a), \text { then } \\
f(t)=\sum_{-\infty}^{\infty} f\left(\frac{n \pi}{a}\right) \frac{\sin (a t-n \pi)}{a t-n \pi} .
\end{gathered}
$$

J. M. Whittaker came very close to touching the question of interpolating functions sampled at nonequidistant points when he considered a more general partial fraction series with samples at $\left\{C_{n}\right\}$, a strictly increasing sequence of positive numbers which is square summable. He noted that his cardinal series and its finite Fourier transform representation did not apply but hinted that a similar theorem would hold if $C_{n}=j_{0, n}$, the $n$th zero of the Bessel function $J_{0}(x)$. So it is no surprise to find Bessel functions among the first examples of the following Kramer sampling theorem [3].

Let $I$ be an interval. Suppose that for each real $t$

$$
f(t)=\int_{I} K(t, x) g(x) d x
$$

where $g(x) \epsilon L^{2}(I)$. Suppose also that for each real $t, K(t, x) \epsilon L^{2}(I)$ and that there exists a countable set $E=\left\{t_{n}\right\}$ such that $\left\{K\left(t_{n}, x\right)\right\}$ is a complete orthogonal set on $L^{2}(I)$. Then

$$
f(t)=\lim _{N \rightarrow \infty} \sum_{n} f<N\left(t_{n}\right) S_{n}(t),
$$

\footnotetext{
**An invited paper.

${ }^{1}$ Figures in brackets indicate the literature references at the end of this paper.
} 
where

$$
S_{n}(t)=\frac{\int_{I} K(t, x) \overline{K\left(t_{n}, x\right) d x}}{\int_{I}\left|K\left(t_{n}, x\right)\right|^{2} d x}
$$

Kramer showed that these conditions on the kernel $K(t, x)$ are satisfied by the solution of any $n$th order, self-adjoint differential equation and gave as illustrations a first-order equation and the Bessel equation. Before Kramer, Weiss [4] had arrived at the same result for $n=2$. Kramer illustrated this theorem for the case of $K(t, x)=J_{m}(x t)$ with the sampling function

$$
S_{n}(t)=\frac{2 j_{m, n} J_{m}(t)}{\left(j_{m, n}^{2}-t^{2}\right)_{m+1}^{J}\left(j_{m, n}\right)}, \quad J_{m}\left(j_{m}, n\right)=0
$$

and Campbell [5] illustrated it for the case of $K(t, x)=P_{t-1 / 2}(x)$, the Legendre function, to obtain

$$
S_{n}(t)=\frac{2 n+1}{\pi} \frac{\sin \left(t-n-\frac{1}{2}\right)}{\left(t-n-\frac{1}{2}\right)\left(t+n+\frac{1}{2}\right)}
$$

In the next section we calculate $S_{n}(t)$ for the case of the associated Legendre functions, the Gegenbauer functions, a Chebyshev function, the prolate spheroidal functions and a Bessel-like function satisfying a fourth-order differential equation. For more details of the calculations one may consult [6].

\section{Further Application of the Kramer Generalized Sampling Theorem}

(i) Associated Legendre functions. Consider the Legendre equation [7, p. 121]:

$$
\left(1-z^{2}\right) \frac{d^{2} u}{d z^{2}}-2 z \frac{d u}{d z}+\left[t(t+1)-\frac{\mu^{2}}{1-z^{2}}\right] u=0
$$

where $z, t, \mu$ are unrestricted. The solution is given by:

$$
u(z)=P \mu(z)=\frac{1}{\Gamma(1-\mu)}\left(\frac{z+1}{z-1}\right)_{2}^{\mu / 2} F_{1}\left(-t, t+1 ; 1-\mu ; \frac{1}{2}-\frac{z}{2}\right),|1-z|<2 .
$$

To obtain the sampling function $S_{n}(t)$ in (5) we need the integral $\int_{-1}^{1} P_{t}^{m}(x) P_{n}^{m}(x) d x$, where $n$ and $m$ are integers and $t$ is unrestricted. We substitute the infinite series expansion for $P_{t}^{m}(x)[7$, p. 167] and integrate term by term to obtain

$$
S_{n}(t)=(-1)^{m} \frac{2 n+1}{\pi} \frac{(n-m) !}{(n+m) !} \frac{\Gamma(t+m+1)}{\Gamma(t-m+1)} \frac{\sin \pi(t-n)}{(t-n)(t+n+1)}
$$

(ii) Gegenbauer functions: Consider the differential equation [7, p. 178]:

$$
\frac{d}{d z}\left[\left(1-z^{2}\right)\left(1-z^{2}\right)^{\nu-1 / 2} \frac{d u}{d z}\right]+\lambda_{t}\left(1-z^{2}\right)^{v-1 / 2} u=0, \lambda_{t}=t(t+2 \nu),
$$

satisfied by the Gegenbauer functions $C_{t}^{\nu}(x)$. For $S_{n}(t)$ in (5) we need $\int_{-1}^{1} C_{t}^{\nu}(x) C_{n}^{\nu}(x)\left(1-x^{2}\right)^{\nu-1 / 2} d x$, $t$ being an unrestricted real parameter. We use [7, p. 159, eq (27), p. 178, and eq (23), p. 140] to obtain

$$
C_{t}^{m+1 / 2}(x)=\frac{2^{m} m !\left(z^{2}-1\right)^{-\frac{m}{2}}}{(2 m) !} P_{t+m}^{m}(x), m+\frac{1}{2}>0 .
$$


If we combine this result with (10) we obtain

$$
\begin{gathered}
S_{n}(t)=\frac{2}{\pi}\left[\frac{2^{2 m} m !}{(2 m) !}\right]^{2} \frac{n !\left(n+m+\frac{1}{2}\right) \Gamma^{2}\left(m+\frac{1}{2}\right) \Gamma(t+2 m+1) \sin \pi(t-n)}{(2 m+n) !(t+n+2 m+1) \Gamma(t+1) \pi(t-n)}, \\
m+\frac{1}{2}>0 .
\end{gathered}
$$

(iii) Chebyshev functions. These are defined as $T_{t}(x)=C_{t}^{0}(x)$ and $U_{t}(x)=C_{t}^{1}(x)$ but we cannot use (13) for either. To treat the latter case we write

$$
C_{t}^{\mu}(x)=\frac{2^{\mu-1 / 2} \Gamma(t+2 \mu) \Gamma\left(\mu+\frac{1}{2}\right)}{\Gamma(2 \mu) \Gamma(t+1)}\left(1-x^{2}\right)^{\frac{1-2 \mu}{4}} P_{t+\mu-1 / 2-1}^{-1 / 2}(x), \quad \operatorname{Re} \mu>0
$$

compare (12), and use [7 p. 169, eq (1)]

$$
\begin{gathered}
\int_{-1}^{1} P_{t}^{\mu}(x) P_{\sigma}^{\mu}(x) d x=\frac{1}{(t-\sigma)(t+\sigma+1)}\left[x(t-\sigma) P_{t}^{\mu}(x) P_{\sigma}^{\mu}(x)+(\sigma+\mu) P_{t}^{\mu}(x) P_{\sigma-1}^{\mu}(x)\right. \\
\left.-(t+\mu) P_{t-1}^{\mu}(x) P_{\sigma}^{\mu}(x)\right]_{-1}^{1}
\end{gathered}
$$

to obtain

$$
\begin{gathered}
\int_{-1}^{1} U_{t}(x) U_{n}(x)\left(1-x^{2}\right)^{1 / 2} d x=\int_{-1}^{1} C_{t}^{1}(x) C_{n}^{1}(x)\left(1-x^{2}\right)^{1 / 2} d x \\
=\frac{1}{(t-n)(t+n+2)} \cdot \frac{2 \Gamma(t+2) \Gamma(n+2)\left[\Gamma\left(\frac{3}{2}\right)\right]^{2}}{[\Gamma(2)]^{2} \Gamma(t+1) n !}\left[x(t-n) P_{t+1 / 2}^{-1 / 2}(x) P_{n+1 / 2}^{-1 / 2}(x)\right. \\
\left.+n P_{t+1 / 2}^{-1 / 2}(x) P_{n-1 / 2}^{-1 / 2}(x)-t P_{t-1 / 2}^{-1 / 2}(x) P_{n+1 / 2}^{-1 / 2}(x)\right]_{-1}^{1} .
\end{gathered}
$$

This result plus the orthogonal property

$$
\int_{-1}^{1}\left\{U_{n}(x)\right\}^{2}\left(1-x^{2}\right)^{1 / 2} d x=\pi
$$

yields the required $S_{n}(t)$.

The result for $T_{t}(x)=C_{t}^{0}(x)$, the Chebyshev function of the first kind is not available, since the above treatment is limited by the condition $\operatorname{Re} \mu>0$ in (14).

(iv) Spheroidal wave functions. Consider the differential equation [8, p. 134, eq (1)]:

$$
\left(1-z^{2}\right) \frac{d^{2} y}{d z^{2}}-2 z \frac{d y}{d z}+\left[\lambda+4 \theta\left(1-z^{2}\right)-\frac{\mu^{2}}{1-z^{2}}\right] y=0
$$

where $\lambda, \theta, \mu$ are given real or complex parameters, $z$ is a complex variable and the eigenvalue $\lambda_{t}^{\mu}(\theta)=t(t+1)$ at $\theta=0$. For $\mu=m$, an integer, a solution of (17) which remains bounded at $z=1$ is the prolate spheroidal function, $P s_{t}^{m}(x, \theta)$. For calculating $S_{n^{\prime}}(t)$ in (5) we need the value of the integral $\int_{-1}^{1} P s_{t}^{m}(x, \theta) P s_{n^{\prime}}^{m}(x, \theta) d x$. First we substitute [8, p. 138, eq (22)],

$$
P s_{t}^{m}(x, \theta)=\sum_{r=-\infty}^{\infty}(-1)^{r} a_{t, r}^{m}(\theta) P_{t+2 r}^{m}(x),
$$


then integrate term by term, using the infinite series expansion for $P_{t+2 r}^{m}(x)$ in terms of $P_{n}^{m}(x)$ $[7$, p. 167] and the result $[8$, p. 158 , eq $(1)]$,

$$
\int_{-1}^{1} P s_{n^{\prime}}^{m}(x) P_{n}^{m}(x) d x=\left\{\begin{array}{l}
(-1)^{r} a_{n^{\prime}, r}^{m}(\theta) \frac{(n+m) !}{\left(n+\frac{1}{2}\right)(n-m) !}, \quad n-n^{\prime}=2 r, \\
0, \quad n-n^{\prime} \text { negative or odd },
\end{array}\right.
$$

to obtain

$$
\int_{-1}^{1} P s_{n^{\prime}}^{m}(x, \theta) P s_{t}^{m}(x, \theta) d x=2 \sum_{r=0}^{\infty} a_{n^{\prime}, r}^{m} a_{t, r}^{m} \frac{\Gamma(t+2 r+m+1) \sin \pi\left(t-n^{\prime}\right)}{\Gamma(t+2 r-m+1)\left(t+n^{\prime}+4 r+1\right) \pi\left(t-n^{\prime}\right)}
$$

We note that this new result reduces to the special case quoted in $[8, \mathrm{p} .147$, eq (6)], since if we let $t=n=n^{\prime}$ in (20) we arrive at

$$
\begin{array}{r}
\left.\int_{-1}^{1} P s_{n^{\prime}}^{m}(x, \theta) P s_{n}^{m} x, \theta\right) d x=\delta_{n n^{\prime}} \sum_{r=0}^{\infty}\left(a_{n^{\prime}}^{m}, r\right)^{2} \frac{\left(n^{\prime}+2 r+m\right) !}{\left(n^{\prime}+2 r-m\right) !} \cdot \frac{1}{n^{\prime}+2 r+\frac{1}{2}}, \\
2 \mathrm{r} \geqslant m-n^{\prime},
\end{array}
$$

and when $[8$, p. 147, eq (7)] is used this becomes

$$
\int_{-1}^{1}\left[P s_{n}^{m}(x)\right]^{2} d x=\frac{1}{n+\frac{1}{2}} \frac{(n+m) !}{(n-m) !}
$$

To evaluate $S_{n}(t)$ in (5) we merely substitute (20) and (22).

(v) Fourth-Order Differential Equations. The following self-adjoint boundary value problem is of interest in signal detection [9]

$$
\begin{gathered}
{\left[D^{4}-\left(m^{2}+\bar{m}^{2}\right) D^{2}+m^{2} \bar{m}^{2}\right] y\left(t e^{-x}\right)=t^{2} e^{-2 x} y\left(t e^{-x}\right) .} \\
y(t)=0, y^{\prime}\left(t_{n}\right)=0, n=1,2, \ldots
\end{gathered}
$$

If we take a new variable $u=e^{-x}$, the solution $H_{m}\left(t_{n} u\right)$ is a Bessel-like function which satisfies Kramer's conditions. To obtain $S_{n}(t)$ for (5) we need the integral $\int_{0}^{1} u H_{m}(t u) H_{m}\left(t_{n} u\right) d u$. For this we consider (23) with $t$ replaced by $t_{n}$, and eliminate the constant coefficient terms between this equation and the original (23) to arrive at

$$
S_{n}(t)=\frac{2 t H_{m}^{\prime}(t)}{\left(t^{2}-t_{n}^{2}\right) H_{m}^{\prime \prime}\left(t_{n}\right)}
$$

\section{Applications in Physics}

The scattering amplitude of two spinless particles interacting via a spherically symmetric potential may be written in the form [10]

$$
A(k, z)=\frac{1}{2 i k} \sum_{l=0}^{\infty}(2 l+1)\left[S_{l}(k)-1\right] P_{l}(z),
$$

where $k$ is the wave number, $z=\cos \theta, P_{l}(x)$ is the Legendre polynomial, $S_{l}$ is the $l$ th eigenvalue of the unitary $S$-matrix, and

$$
S_{l}-1=i k \int_{-1}^{1} A(k, z) P_{l}(z) d z
$$


When the $A(k, z)$ of (25) is represented by the Watson transform as a contour integral in the $\lambda$-plane, where $\lambda$ represents the complex angular momentum, it is found that the contribution to $A(k, z)$ from the $n$th Regge pole of the $S$-matrix at $\lambda=\alpha_{n}$ with residue $\beta_{n}$ is

$$
\frac{i \pi}{k}\left(\alpha_{n}+\frac{1}{2}\right) \beta_{n} P_{\alpha_{n}}(-z) / \sin \left(\pi \alpha_{n}\right) .
$$

To obtain the $S_{l^{(n)}}-1$ corresponding to this term, eq (26) may be used. Hence we need to evaluate the integral $\int_{-1}^{1} P_{\alpha_{n}}(-z) P_{l}(z) d z$, which is the special case of (5) that corresponds to the Legendre function $P_{\alpha_{n}}(x)=K\left(\alpha_{n}, x\right)$. This integral is given in (7) and was used in [10] to give

$$
S^{(n)}-1=\frac{\beta_{n}\left(2 \alpha_{n}+1\right)}{\left(l-\alpha_{n}\right)\left(l+\alpha_{n}+1\right)} .
$$

We point out that at times it is necessary to obtain series expansions for $A(k, z)$ in terms of other orthogonal functions such as the associated Legendre polynomials, the Gegenbauer functions, the Chebyshev polynomials, the prolate spheroidal functions and the Bessel-like functions. In such cases a Watson-like transform corresponding to each of these orthogonal functions may be defined in a similar way. Furthermore, for each of these transforms, the $S_{\rangle^{n}}-1$ term corresponding to the $n$th Regge pole contribution to $A(k, z)$ may be obtained in a way similar to that used for (28). This calculation may be carried out by using the sampling functions corresponding to the orthogonal functions in the kernel of the transforms. These were derived in (10), (13), (16), (20), and (24).

\section{References}

[1] Whittaker, J. M., The "Fourier" theory of the cardinal functions. Proc. Edinburgh. Math. Soc. 1, $169-176$ (1929).

[2] Shannon, C. E., Communications in the Presence of Noise, Proc. I.R.E., 37, 10-12 (1949).

[3] Kramer, H. P., A Generalized Sampling Theorem, J. Math. Phys. 38, 68-72 (1959).

[4] Weiss, P., Sampling theorems associated with Sturm-Liouville systems, Bull. Math. Soc, 63 (1957).

[5] Campbell, L. L., A comparison of the sampling theorems of Kramer and Whittaker, Soc. of Indust. Appl. Math. 12, 117-130 (1964)

[6] Jerri, A. J., On Extension of the Generalized Sampling Theorem Ph.D. Thesis (Tech. Report No. 31) Dept. of Mathematics, Oregon State University, Corvallis, Oregon, June 1967.

[7] Erdélyi, A., et al., Higher Transcendental Functions, I (McGraw-Hill Publishing Company, New York 1953)

[8] Erdélyi. A., et al., Higher Transcendental Functions, III (McGraw-Hill Publishing Company, New York 1953).

[9] Stone, W. M., et al. On the First Probability of Detection Filter, Seattle 1955, p. 31 (Boeing Document, Number D-16921) .

[10] Newton, R. G., The Complex j-Plane (Benjamin, Inc., New York 1964).

(Paper 73B3-304) 